\title{
Sponge-like nanostructured silicon for integrated emitters
}

\author{
A. Hubarevich ${ }^{1}$, P. Jaguiro ${ }^{1}$, Y. Mukha ${ }^{1}$, A. Smirnov ${ }^{1}$, Ya. Solovjov ${ }^{2}$ \\ ${ }^{1}$ Belarusian State University of Informatics and Radioelectronics, Laboratory of Information Displays \\ 6, P. Brovki str., 220013 Minsk, Republic of Belarus \\ ${ }^{2}$ SOE “Transistor Factory”, 16, Korzhenevskogo str., 220108 Minsk, Republic of Belarus
}

\begin{abstract}
A new approach to nanoporous silicon formation is proposed. Anomalies both in low current densities and low fluorine ion concentrations, which is lead to low uniformity of formed porous silicon, are under consideration. It is shown that at very low current densities and fluorine ion concentration high uniformity, high porosity nanoporous silicon layers can be created. Structural, electrical and optical properties of porous silicon formed in a wide range of current densities, doping levels of silicon substrates and fluorine concentrations are presented.
\end{abstract}

Keywords: porous silicon, nanostructure, electroluminescence.

Manuscript received 18.12.09; accepted for publication 08.07.10; published online 30.09.10.

\section{Introduction}

Silicon is an essential foundation in today's microelectronics because of its extraordinary physicochemical, electronic and technological properties. However, because of its indirect bandgap structure monocrystalline silicon cannot be used as material for light emission. At first porous self-organized structure on silicon was created in 1956 at Bell Laboratory. Porous silicon was widely used in microelectronics: IPOS and FIPOS processes, SOI wafers production. In 1990 the quantum effects and room temperature visible luminescence was demonstrated in porous silicon $[1,2]$. This feature awoke researchers' interest, and the first light emission silicon diode appeared some time later. Our group works over avalanche type reverse biased Schottky diodes and its microdisplays as well as optoelectronics applications [35]. Standard technological parameters for formation a high porosity nanostructured $\mathrm{Si}$ are high current densities and high concentrations of hydrofluoric acid. These regimes are not convenient, because of very short process times (some seconds for thin layers) and toxic (high HF vapor pressure), aggressive reagents (etching Al layers and interconnections) are used. However, changing to lower current densities and concentrations leads to instability and low uniformity of the process.

In this paper, we analyze reasons of the instability and propose a new stable and convenient technological regime for high porosity nanostructured $\mathrm{Si}$ formation at ultra small current density and fluorine ions concentration.

\section{Porous silicon formation}

There are three well known regimes corresponding to the areas in the current density - hydrofluoric acid concentration plot, which are illustrated in Fig. 1 [6]. At high densities-concentrations electro-polishing takes place, at low densities-concentrations we have porous silicon formation, and in the middle we deal with a transient regime. Rectangular in the plot shows standard good reproducible, well known regimes [7], while ellipse corresponds to low uniformity processes. The area at the bottom left corner isn't methodically investigated. One of the reasons of this is low buffer capacity of very diluted hydrofluoric acid and chemical changes during the anodizing process. To avoid from "diluted" problem using of salts of hydrofluoric acid in combination with an acid with high buffer capacity is proposed.

The special case at zero current at acidified $\mathrm{NH}_{4} \mathrm{HF}_{2}$ is investigated $[8,9]$, but it doesn't provide enough control on porous silicon morphology. To avoid aggressive etching of Al by HF solutions (up to $4000 \mathrm{~nm}$ per hour), it was proposed to use $14 \mathrm{wt} . \%$ ammonium fluoride, $33 \mathrm{wt} \%$ acetic acid in water ( $30 \mathrm{~nm}$ per hour) as electrolyte for anodizing [10]. However, at current densities $5-80 \mathrm{~mA} / \mathrm{cm}^{2}$ the uniformity is far from perfect.

Note that HF vapor pressure over weak acidified fluoride salts is significantly lower than over hydrofluoric acid solutions. It is strong anomaly in fluorine ions and its derivatives concentration, extrema appear on its dependence of the fluoride salt concentration [11]. 


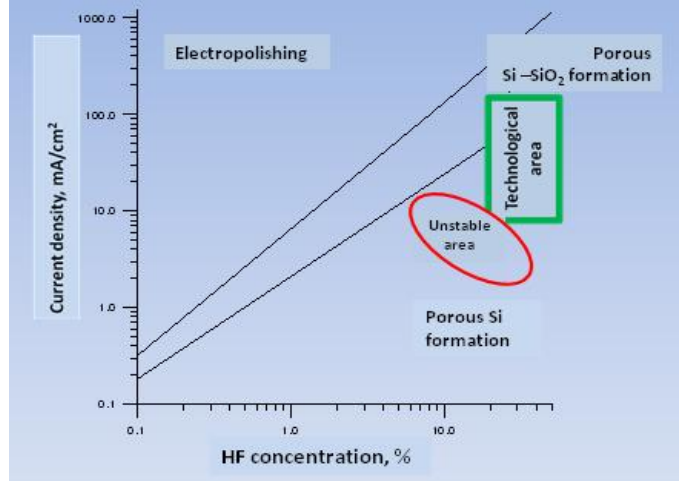

Fig. 1. Different types of porous silicon formation.

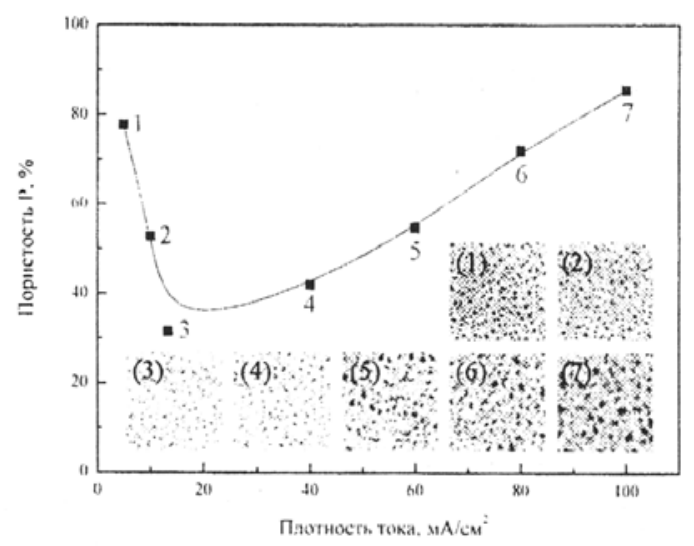

Fig. 2. Porous structure versus current density [12].

Heavy negative doped monocrystalline silicon is used as precursor for nanostructured Schottky junction fabrication, so this material is under our investigation. In Fig. 2 change in the porous structure versus anodizing current density for heavy antimony doped silicon $(0.01 \mathrm{Ohm} \cdot \mathrm{cm}, \quad\langle 100\rangle \quad$ orientation $)$ in $1: 2: 1$ $\mathrm{HF}: \mathrm{C}_{2} \mathrm{H}_{5} \mathrm{OH}: \mathrm{H}_{2} \mathrm{O}$ is presented [12]. Porosity anomaly versus current density is absolutely evident. At about $10 \mathrm{~mA} / \mathrm{cm}^{2}$ the plot turns, and morphology of porous silicon changes from regular vertical holes (at higher current densities) to sponge structure (at lower current densities).

Numeric analysis that was performed by our group earlier $[13,14]$ shows that these changes of porosity can be related to increase of the electrochemical diffusion layer (from 30 to about $500 \mu \mathrm{m}$ ) while the current density slows down. This increase is determined by break off solution agitate by emitted gas bubbles. At low current densities, issue of emitted gas is small, and all hydrogen gas can be dissolved in solution and doesn't form a bubble. The critical current is $4.3 \mathrm{~mA} / \mathrm{cm}^{2}$ for water at ambient conditions. Anodizing process can be unstable near this value $\left(1-10 \mathrm{~mA} / \mathrm{cm}^{2}\right)$, because of local gas bubble agitating and corresponding irregular current distribution. Note that very high porosities can be achieved at low current densities.

\section{Experimental details}

Addition of ethanol to solution allows moistening hydrophobic silicon surface and getting more reproducible results. In our system, it is not necessary to add a big amount of water, because we work at low fluorine ion concentrations and weak dissociated acids. So, the simplest solution is about $4 \%$ of water that is present in rectified ethanol. It is practically impossible to predict ion concentrations in the solution due to the "concentration anomaly" and usage of organic solvent. In this case, we should to carry out more experiments.

N-type $\langle 100\rangle$ oriented phosphorous doped silicon substrates $(0.1 \mathrm{Ohm} \cdot \mathrm{cm}$ and $0.01 \mathrm{Ohm} \cdot \mathrm{cm})$ were used. The samples were anodized in ambient conditions with halogen lamp illumination. Current densities and electrolyte concentrations in rectified ethanol are summarized in Table.

Table. The range of anodization parameters.

\begin{tabular}{|c|c|c|}
\hline $\mathrm{NH}_{4} \mathrm{~F}: \mathrm{H}_{3} \mathrm{PO}_{4}: \mathrm{C}_{2} \mathrm{H}_{5} \mathrm{OH}$ & Current density & Doping level \\
\hline $5-25 \% \mathrm{H}_{3} \mathrm{PO}_{4}$ & $\begin{array}{l}0.01-1 \\
\mathrm{~mA} / \mathrm{cm}^{2}\end{array}$ & $10^{21}-10^{20}$ \\
\hline
\end{tabular}

Pores sizes and structure of PS were observed using the scanning electron microscope (LEO 1550 Gemini). The thickness and porosity of the layers were measured by Spectroscopic Ellipsometer VB-250 and calculated then as describing. Photoluminescence and electroluminescence were measured using spectrometer, photoluminescence was excited by the discrete like of a mercury lamp $330 \mathrm{~nm}$. For electroluminescent measurements $0.7 \mu \mathrm{m}$ Al was PVD and anodized through photoresist mask. $200 \times 200 \mu \mathrm{m}$ pads leaves on the porous silicon layer. Electrical measurements of the Schottky diodes were carried out using a special equipment.

\section{Results and discussion}

In Fig. 3, voltage versus time curves are presented for different samples after anodization.

In Fig. 4, photoluminescence spectra for two samples of PS and monocrystalline silicon are presented.

Figs 5 and 6 shows the structure of PS. Pores sizes are 10 to $20 \mathrm{~nm}$ and porosity reaches $55 \%$ at the current density $0.25 \mathrm{~mA} / \mathrm{cm}^{2}$ and $75 \%$ at the current density $0.025 \mathrm{~mA} / \mathrm{cm}^{2}$. Thus, in the regime of small concentration of $\mathrm{F}^{-}$ions the drop in the current density increases porosity.

Fig. 6 shows the thickness of PS. It is $50 \mathrm{~nm}$ at the current density $0.1 \mathrm{~mA} / \mathrm{cm}^{2}$, time $500 \mathrm{~s}$ and solution $5 \% \mathrm{NH}_{4} \mathrm{~F}: 15 \% \mathrm{H}_{3} \mathrm{PO}_{4}: \mathrm{C}_{2} \mathrm{H}_{5} \mathrm{OH}$.

The structures in Figs 5 and 6 are sponge-like. Sponge forms with porosity $70 \%$ and more can be used in optical applications. 


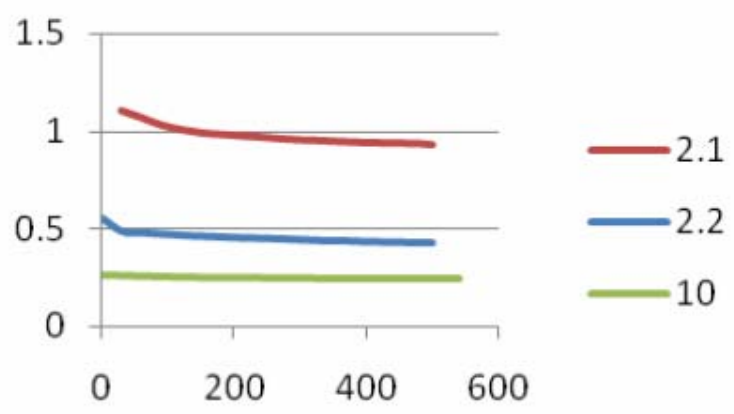

Fig. 3. Voltage versus time dependences.

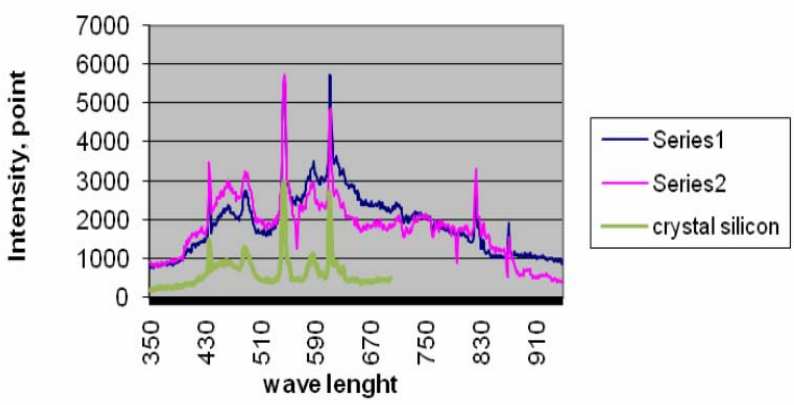

Fig. 4. Photoluminescence spectra.

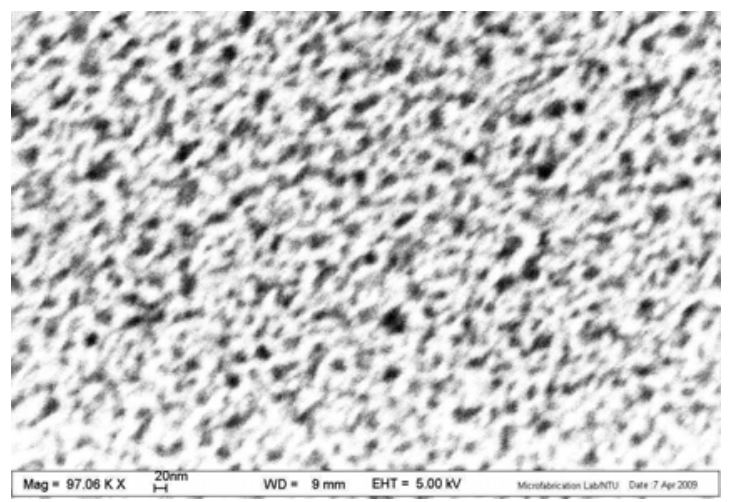

Fig. 5. The structure of PS anodized at $0.25 \mathrm{~mA} / \mathrm{cm}^{2}$ and $5 \%$ $\mathrm{NH}_{4} \mathrm{~F}: 5 \% \mathrm{H}_{3} \mathrm{PO}_{4}: \mathrm{C}_{2} \mathrm{H}_{5} \mathrm{OH}$.

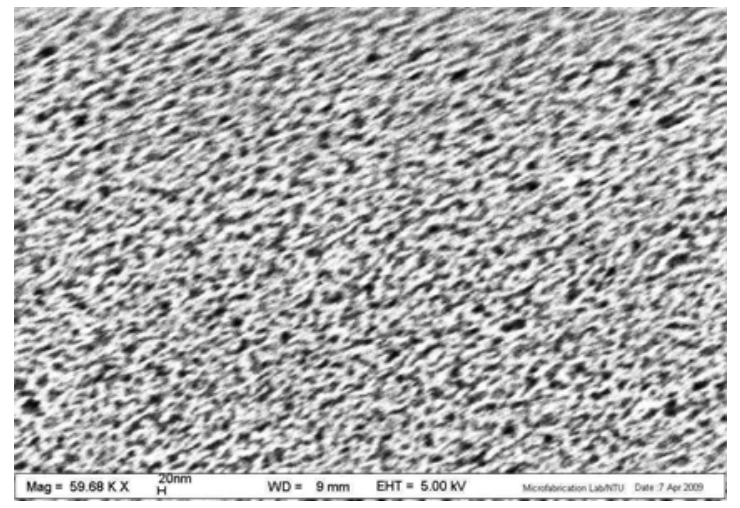

Fig. 6. The structure of PS anodized at $0.025 \mathrm{~mA} / \mathrm{cm}^{2}$ and $5 \% \mathrm{NH}_{4} \mathrm{~F}: 5 \% \mathrm{H}_{3} \mathrm{PO}_{4}: \mathrm{C}_{2} \mathrm{H}_{5} \mathrm{OH}$.

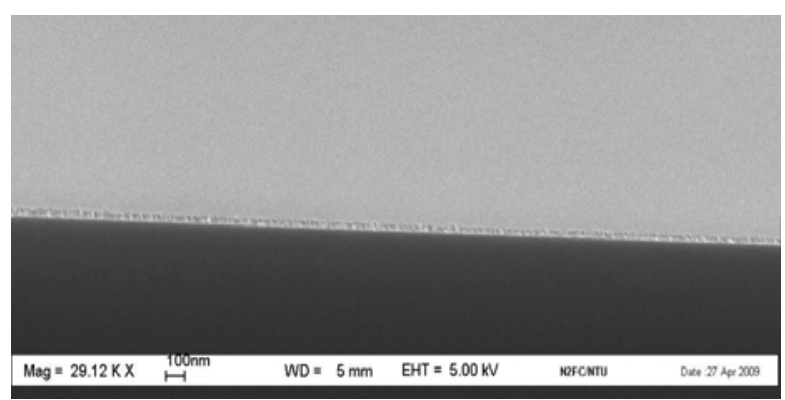

Fig. 7. Cross-section of PS structure at $0.1 \mathrm{~mA} / \mathrm{cm}^{2}, 500 \mathrm{~s}$ and $5 \% \mathrm{NH}_{4} \mathrm{~F}: 15 \% \mathrm{H}_{3} \mathrm{PO}_{4}: \mathrm{C}_{2} \mathrm{H}_{5} \mathrm{OH}$.

\section{Conclusion}

In this work, we report the stable and reproducible regime to produce porous silicon layers at ultra-small current densities and fluorine ion concentrations. The porous silicon structure is of high porosity and spongelike, with a small thickness and allows using it as light emitters, specifically in emission displays.

\section{References}

1. L. Canham, Silicon quantum wire array fabrication by electrochemical and chemical dissolution of wafers // Appl. Phys. Lett. 57, p. 1046 (1990).

2. A.G. Cullis and L.T. Canham, Visible light emission due to quantum size effects in highly porous crystalline silicon // Nature 353, p. 335-338 (1991).

3. S. Lazarouk, P. Jaguiro, S. Katsouba, G. Masini, S. La Monica, G. Maiello and A. Ferrari, Stable electroluminescence from reverse biased n-type porous silicon-aluminum Schottky junction device // Appl. Phys. Lett. 68, p. 1646-1648 (1996).

4. P. Jaguiro, P. Katsuba, S. Lazarouk, M. Farmer and A. Smirnov, Si-based emissive microdisplays // Physica E: Low-dimensional Systems and Nanostructures 41, p. 927-930 (2009).

5. P. Jaguiro, P. Katsuba, S. Lazarouk, S. Moore and A. Smirnov, Si-based optoelectronic couple // Physica E: Low-dimensional Systems and Nanostructures 41, p. 1094-1096 (2009).

6. X. Zhang, S. Collins and R. Smith, Porous silicon formation and electropolishing of silicon by anodic polarization in HF solution // J. Electrochem. Soc. 136, p. 1561 (1989).

7. G. Sperveslage, J. Grobe, G. Egbers and A. Benninghoven, Porous silicon: repeatability of generation? // Fresenius' J. Analyt. Chem. 361, p. 554-557 (1998).

8. K.W. Kolasinski, Silicon nanostructures from electroless electrochemical etching // Current Opinion in Solid State and Materials Science 9, p. 73-83 (2005). 
9. M. Nahidi and K.W. Kolasinski, Effects of stain etchant composition on the photoluminescence and morphology of porous silicon // J. Electrochem. Soc. 153, p. C19-C26 (2006).

10. M. Kuhl, G. O'Halloran, P. Gennissen and P. French, Formation of porous silicon using an ammonium fluoride based electrolyte for application as a sacrificial layer // J. Micromechanics and Microeng. 8, p. 317-322 (1998).

11. K.W. Kolasinski, The composition of fluoride solutions // J. Electrochem. Soc. 152, p. J99-J104 (2005).
12. K. Kholostov, O. Filatova and V. Bondarenko, Investigation of structure of mesoporous silicon // Doklady Belorus. Gosudarstv.Universiteta Inform. Radioelektroniki 35, p. $72-76$ (2008).

13. S. LaMonica, P. Jaguiro and A. Ferrari, A thermodynamical explanation for pore growing stability in porous silicon // Intern. Symp. on Pits and Pores, at the 191st Meeting of the Electrochem. Soc., Montreal, Canada, 1997.

14. P. Jaguiro, S. LaMonica, S. Lazarouk and A. Ferrari, Theoretical model of porous silicon formation // Intern. Symp. on Pits and Pores, at the 191st Meeting of the Electrochem. Soc., Montreal, Canada, 1997. 